be integrated with specialty care to provide optimal clinical management for people living with HIV.

Objective To examine the effectiveness of shared care models of HIV between primary care and specialty care and how primary care providers can assist in improving the care of people with HIV.

Methods Three databases, PubMed, Medline and EMBase were searched for relevant terms from studies published in the period from 1996 to 2011. Studies were included that integrated primary care in HIV management and included highly active antiretroviral therapy (HAART) as part of the treatment modality.

Results 11 studies that met the inclusion criteria were included in this review. Primary care was found to be at least as effective in HIV counselling, testing and treatment and, to a lesser degree, prevention, when compared to specialty care alone. Screening for HIV at a primary care level was cost-effective, especially in a high HIV prevalence and high-risk community. There were no significant adverse clinical outcomes reported in a primary care approach. Effectiveness of various interventions using a primary care approach were demonstrated in the review, including HAART adherence programmes, home care, the involvement of peer health workers and perinatal use of HAART.

Conclusions Primary care has an important role in the shared care of the diagnosis and management of people with HIV. Some improvements with current guidelines on the management in primary care of people with HIV in developing countries should be considered.

\section{P115 LEARNING FROM PATIENTS TO REDESIGN AN HIV SERVICE}

doi:10.1136/sextrans-2012-050601c.115

S Kegg, * S Goddard, J Russell. South London Healthcare NHS Trust

Background/Aims Our HIV cohort more than doubled between $2004 / 11$ with no increase in clinic capacity or staffing. Some of our service users have complex health needs-related to delayed HIV diagnosis, poor adherence to ART and co-morbidities-but an increasing number are stable on treatment and more than half have their medication delivered to their home. We are reviewing our service model to focus on the needs of our complex patients but at the same time providing a responsive, quality service to the stable patients. A key part of this review is to elicit our patients' views on the current service and how it might be improved.

Methods A self-administered questionnaire completed by $\mathrm{HIV}+$ service users.

Results 50 people completed a questionnaire and $76 \%$ described their current health as good to excellent. $43 \%$ were attending appointments 3-4 monthly, with 39\% attending more frequently. $72 \%$ wished to continue to be seen at the same frequency. Most (76\%) reported that appointments were available at suitable times. Barriers to clinic attendance included poor car-parking (44\%) and lack of transport links (13\%). A majority (84\%) have access to a personal computer and $53 \%$ have a webcam-of these $50 \%$ would value an online consultation. $32 \%$ would consider HIV monitoring by their GP and 33\% would like to receive some of their care in this setting. Of the people currently receiving ART delivered to their home $35 \%$ would like monitoring to be performed by the individual delivering the medications.

Conclusions Most patients are satisfied with the current model of care and may be reluctant to be seen less often. However there is a softening in the reluctance to involve GPs in HIV care and a willingness to consider models using newer technologies and care at home. We have reviewed our clinic template increasing the number of early and late appointments and are actively developing pilots to explore new ways to deliver care to increase choice and flexibility for our patients.

\section{P116 \\ ASSESSING THE SEXUAL VIOLENCE SERVICES CURRENTLY PROVIDED IN GENITOURINARY MEDICINE CLINICS}

doi:10.1136/sextrans-2012-050601c.116

${ }^{1} \mathrm{C}$ R Emerson, ${ }^{*} \mathrm{R}$ Sacks, ${ }^{3} \mathrm{G}$ E Foster. ${ }^{1}$ Belfast Trust; ${ }^{2}$ Imperial College Healthcare NHS Trust, ${ }^{3}$ Barts and the London

Background Many patients present to Genitourinary medicine (GUM) clinics following sexual violence (SV) occurring in circumstances including sexual assault (SA), domestic violence (DV), sex work (SW) and trafficking. BASHH has guidelines for SA but not for addressing SV in other situations. Are GUM clinics providing services for these needs?

Aims To assess services currently available in GUM for those disclosing SV.

Methods A cross sectional anonymous online survey of UK GUM clinics was performed. The survey was designed by the authors and piloted. Information was gathered on specific clinics for SV, how often they occur and which members of the multidisciplinary team are involved, and training available for SV. Links with other support services was ascertained. The data were analysed using Microsoft Excel. Results The response rate was 66/178 (37\%), with all regions and deaneries being represented. All responding clinics provide PEP, emergency contraception, STI screen, hepatitis prophylaxis and advice for those presenting after SA. $24 \%$ of clinics never use chain of evidence procedures. $80 \%$ have a local sexual assault referral centre (of these $87 \%$ were happy with this service). $80 \%, 77 \%, 50 \%$ and $38 \%$ have no dedicated clinic for female genital mutilation, sexual trafficking, DV and SW respectively and less than $50 \%$ have local training or procedures for these facets. $32 \%$ ask about nonconsensual sex in all GUM consults, 53\% ask this in special groups only. $47 \%$ routinely ask about SW.

Discussion There is a wide variation in the SV services available at GUM clinics. Sexual assault services meet guideline recommendations for GUM but SV occurring in other contexts may not be addressed. Sexual violence is increasingly reported and GUM clinics may be the first or only location of presentation. We recommend further training and guidelines to ensure clinics are equipped to meet this need.

\section{P117 AUDIT ON UPTAKE OF HIV TEST IN SEXUAL HEALTH CLINIC, ANTENATAL AND TOP SERVICES IN DUMFRIES BETWEEN JANUARY AND JUNE 2011}

doi:10.1136/sextrans-2012-050601c.117

0 Steshenko.* Dumfries and Galloway Royal Infirmary

Background BASHH introduced "UK National Guideline for HIV testing 2008" recommending universal HIV testing in GUM or sexual health clinics, antenatal and termination of pregnancy services. Early identification HIV positive persons help to reduce further transmission of HIV infection to others and allow referring them to appropriate services for further treatment.

Aims and Objectives The audit was undertaken to identify compliance of local sexual health clinic, antenatal and TOP services with "UK National Guideline for HIV testing 2008". To establish uptake of HIV test by patients accessing Sexual Health Clinic, Antenatal and TOP services in Dumfries between 1st January and 30th June 2011 and give recommendations according to findings.

Methods Retrospective audit of electronic patients' records of all persons accessing the local sexual health clinic in relation to STI issues, pregnant women attending antenatal clinic, patients receiving TOP service care between 1st January and 30th June 2011. Results From 844 episodes of care in sexual health clinic, HIV test performed in 400 (47\%) episodes. 511 women attended antenatal 